\title{
SIMULTANEOUS APPROXIMATION FOR THE PHILLIPS OPERATORS
}

\author{
N. K. GOVIL, VIJAY GUPTA, AND MUHAMMAD ASLAM NOOR
}

Received 18 October 2005; Accepted 1 March 2006

We study the simultaneous approximation properties of the well-known Phillips operators. We establish the rate of convergence of the Phillips operators in simultaneous approximation by means of the decomposition technique for functions of bounded variation.

Copyright (c) 2006 Hindawi Publishing Corporation. All rights reserved.

\section{Introduction}

Phillips operators (see, e.g., [5]) are defined as

$$
P_{n}(f, x)=n \sum_{k=1}^{\infty} p_{n, k}(x) \int_{0}^{\infty} p_{n, k-1}(t) f(t) d t+e^{-n x} f(0), \quad n \in N, x \in[0, \infty)
$$

where

$$
p_{n, k}(x)=e^{-n x} \frac{(n x)^{k}}{k !}
$$

May [4] estimated some direct and inverse results for certain combinations of these operators. Very recently Finta and Gupta [1] studied some direct and inverse results for the second-order Ditzian-Totik modulus of smoothness. The rates of convergence in ordinary approximation for function of bounded variation for these operators and similar types of operators were estimated in $[2,3,7]$. Very recently Srivastava and Gupta [6] proposed a general family of linear positive operators, which include the Phillips operators as special case, but they have estimated the rate of convergence in ordinary approximation. In the present paper we investigate and estimate the rate of convergence of the Phillips operators in simultaneous approximation by means of the decomposition technique for functions of bounded variation. 
2 Simultaneous approximation for the Phillips operators

\section{Auxiliary results}

In this section we give the following lemmas, which will be needed to prove our main result, given in Section 3.

Lemma 2.1. For all $x \in(0, \infty)$ and $k \in N \cup\{0\}$,

$$
p_{n, k}(x) \leq \frac{1}{\sqrt{2 e n x}}
$$

where the constant $1 / \sqrt{2 e}$ and the estimation order $n^{-1 / 2}($ for $n \rightarrow \infty)$ are the best possible. Proof. Following [8], we have

$$
p_{n, k}(x) \leq \frac{H(j)}{\sqrt{n x}}, \quad k \geq j
$$

where $H(j)=(j+1 / 2)^{j+1 / 2} e^{-(j+1 / 2)} / j$ !.

Since $\max _{j \geq 0} H(j)=H(0)=1 / \sqrt{2 e}$, it follows that

$$
p_{n, k}(x) \leq \frac{1}{\sqrt{2 e n x}} \text { for each integer } k \geq 0,
$$

and Lemma 2.1 is thus proved.

Remark 2.2. The above lemma can be utilized to give better estimate over the main results of $[2,3,6]$.

Lemma 2.3. If $f \in L_{1}[0, \infty), f^{(r-1)} \in A \cdot C \cdot{ }_{\text {loc }}, r \in N$, and $f^{(r)} \in L_{1}[0, \infty)$, then

$$
P_{n}^{(r)}(f, x)=n \sum_{k=0}^{\infty} p_{n, k}(x) \int_{0}^{\infty} p_{n, k+r-1}(t) f^{(r)}(t) d t .
$$

Proof. First by the definition,

$$
P_{n}^{(1)}(f, x)=n \sum_{k=1}^{\infty} p_{n, k}^{(1)}(x) \int_{0}^{\infty} p_{n, k-1}(t) f(t) d t-n \cdot e^{-n x} f(0) .
$$

Using the identity $p_{n, k}^{(1)}(x)=n\left[p_{n, k-1}(x)-p_{n, k}(x)\right], k \geq 1$, we have

$$
\begin{aligned}
P_{n}^{(1)}(f, x)= & n \sum_{k=1}^{\infty} n\left[p_{n, k-1}(x)-p_{n, k}(x)\right] \int_{0}^{\infty} p_{n, k-1}(t) f(t) d t-n \cdot e^{-n x} f(0) \\
= & n^{2} p_{n, 0}(x) \int_{0}^{\infty} p_{n, 0}(t) f(t) d t-n e^{-n x} f(0) \\
& +n^{2} \sum_{k=1}^{\infty} p_{n, k}(x) \int_{0}^{\infty}\left[p_{n, k}(t)-p_{n, k-1}(t)\right] f(t) d t \\
= & n^{2} e^{-n x} \int_{0}^{\infty} e^{-n t} f(t) d t+n^{2} \sum_{k=1}^{\infty} p_{n, k}(x) \int_{0}^{\infty} \frac{-1}{n} p_{n, k}^{(1)}(t) f(t) d t-n e^{-n x} f(0)
\end{aligned}
$$


N. K. Govil et al. 3

$$
\begin{aligned}
= & n^{2} e^{-n x}\left[\left.f(t) \frac{e^{-n t}}{-n}\right|_{0} ^{\infty}-\int_{0}^{\infty} f^{(1)}(t) \frac{e^{-n t}}{-n} d t\right] \\
& -n \sum_{k=1}^{\infty} p_{n, k}(x)\left[\left.f(t) p_{n, k}(t)\right|_{0} ^{\infty}-\int_{0}^{\infty} p_{n, k}(t) f^{(1)}(t) d t\right]-n e^{-n x} f(0) \\
= & n e^{-n x} \int_{0}^{\infty} e^{-n t} f^{(1)}(t) d t+n \sum_{k=1}^{\infty} p_{n, k}(x) \int_{0}^{\infty} p_{n, k}(t) f^{(1)}(t) d t \\
= & n \sum_{k=0}^{\infty} p_{n, k}(x) \int_{0}^{\infty} p_{n, k}(t) f^{(1)}(t) d t .
\end{aligned}
$$

Thus the result is true for $r=1$. We prove the result by induction hypothesis, and for this we suppose it is true for $r=i$. Then

$$
P_{n}^{(i)}(f, x)=n \sum_{k=0}^{\infty} p_{n, k}(x) \int_{0}^{\infty} p_{n, k+i-1}(t) f^{(i)}(t) d t .
$$

Again using the identity $p_{n, k}^{(1)}(x)=n\left[p_{n, k-1}(x)-p_{n, k}(x)\right], k \geq 1$, it follows that

$$
\begin{aligned}
P_{n}^{(i+1)}(f, x)= & n \sum_{k=0}^{\infty} n\left[p_{n, k-1}(x)-p_{n, k}(x)\right] \int_{0}^{\infty} p_{n, k+i-1}(t) f^{(i)}(t) d t \\
& +n\left(-n e^{-n x}\right) \int_{0}^{\infty} p_{n, i-1}(t) f^{(i)}(t) d t \\
= & n^{2} p_{n, 0}(x) \int_{0}^{\infty} p_{n, i}(t) f^{(i)}(t) d t-n^{2} p_{n, 0}(x) \int_{0}^{\infty} p_{n, i-1}(t) f^{(i)}(t) d t \\
& +n^{2} \sum_{k=1}^{\infty} p_{n, k}(x) \int_{0}^{\infty}\left[p_{n, k+i}(t)-p_{n, k+i-1}(t)\right] f^{(i)}(t) d t \\
= & n^{2} p_{n, 0}(x) \int_{0}^{\infty}\left(\frac{-p_{n, i}^{(1)}(t)}{n}\right) f^{(i)}(t) d t+n^{2} \sum_{k=1}^{\infty} p_{n, k}(x) \int_{0}^{\infty}\left(\frac{-p_{n, k+i}^{(1)}(t)}{n}\right) f^{(i)}(t) d t .
\end{aligned}
$$

Integrating by parts, we obtain

$$
P_{n}^{(i+1)}(f, x)=n \sum_{k=0}^{\infty} p_{n, k}(x) \int_{0}^{\infty} p_{n, k+i}(t) f^{(i+1)}(t) d t,
$$

which was proved and this completes the proof of Lemma 2.3.

Lemma 2.4. For $m \in N \cup\{0\}, r \in N$, if the mth-order moment is defined by

$$
\mu_{r, n, m}(x)=n \sum_{k=0}^{\infty} p_{n, k}(x) \int_{0}^{\infty} p_{n, k+r-1}(t)(t-x)^{m} d t,
$$


4 Simultaneous approximation for the Phillips operators

then

$$
\mu_{r, n, 0}(x)=1, \quad \mu_{r, n, 1}(x)=\frac{r}{n}, \quad \mu_{r, n, 2}(x)=\frac{2 n x+r(1+r)}{n^{2}} .
$$

Also, there holds the following recurrence relation:

$$
n \mu_{r, n, m+1}(x)=x\left[\mu_{r, n, m}^{(1)}(x)+2 m \mu_{r, n, m-1}(x)\right]+(m+r) \mu_{r, n, m}(x) .
$$

Consequently, by the recurrence relation, for all $x \in[0, \infty)$,

$$
\mu_{r, n, m}(x)=O\left(n^{-[(m+1) / 2]}\right)
$$

Proof. Using the identity $x p_{n, k}^{\prime}(x)=(k-n x) p_{n, k}(x)$, we have

$$
x \mu_{r, n, m}^{(1)}(x)=n \sum_{k=0}^{\infty} x p_{n, k}^{\prime}(x) \int_{0}^{\infty} p_{n, k+r-1}(t)(t-x)^{m} d t-m x \mu_{r, n, m-1}(x) .
$$

Thus

$$
\begin{aligned}
x\left[\mu_{r, n, m}^{(1)}(x)+m \mu_{r, n, m-1}(x)\right] & \\
= & n \sum_{k=0}^{\infty} p_{n, k}(x) \int_{0}^{\infty}[\{(k+r-1)-n t\}+n(t-x)+1-r] p_{n, k+r-1}(t)(t-x)^{m} d t \\
= & n \sum_{k=0}^{\infty} p_{n, k}(x) \int_{0}^{\infty} t p_{n, k+r-1}^{\prime}(t)(t-x)^{m} d t+n \mu_{r, n, m+1}(x)+(1-r) \mu_{r, n, m}(x) \\
= & n \sum_{k=0}^{\infty} p_{n, k}(x) \int_{0}^{\infty} p_{n, k+r-1}^{\prime}(t)(t-x)^{m+1} d t \\
& +n x \sum_{k=0}^{\infty} p_{n, k}(x) \int_{0}^{\infty} p_{n, k+r-1}^{\prime}(t)(t-x)^{m} d t+n \mu_{r, n, m+1}(x)+(1-r) \mu_{r, n, m}(x) .
\end{aligned}
$$

Integrating by parts, we get the required recurrence relation. The other consequences easily follow from the recurrence relation.

Remark 2.5. In particular by Lemma 2.4, for given any number $\lambda>2$ and $0<x<\infty$, we get for $n \geq 2$,

$$
\mu_{r, n, 2}(x) \leq \frac{\lambda x}{n}
$$

Remark 2.6. We can observe from Lemmas 2.3 and 2.4 that for $r=0$, the summation over $k$ starts from 1. For $r=0$, Lemma 2.4 may be defined as in [6, Lemma 2], with $c=0$. 
Lemma 2.7. Suppose $x \in(0, \infty), r \in N$, and $K_{r, n}(x, t)=n \sum_{k=0}^{\infty} p_{n, k}(x) p_{n, k+r-1}(t)$. Then for $\lambda>2$ and for $n \geq 2$, there hold

$$
\begin{array}{ll}
\int_{0}^{y} K_{r, n}(x, t) d t \leq \frac{\lambda x}{n(x-y)^{2}}, & 0 \leq y<x, \\
\int_{z}^{\infty} K_{r, n}(x, t) d t \leq \frac{\lambda x}{n(z-x)^{2}}, & x<z<\infty .
\end{array}
$$

Proof. We first prove (2.17) as follows:

$$
\begin{aligned}
\int_{0}^{y} K_{r, n}(x, t) d t & \leq \int_{0}^{y} \frac{(x-t)^{2}}{(x-y)^{2}} K_{r, n}(x, t) d t \\
& \leq \frac{1}{(x-y)^{2}} P_{n}\left((t-x)^{2}, x\right) \leq \frac{\mu_{r, n, 2}(x)}{(x-y)^{2}} \leq \frac{\lambda x}{n(x-y)^{2}}
\end{aligned}
$$

by using (2.16). The proof of (2.18) follows along similar lines.

\section{Rate of convergence}

We denote the class $B_{r, \alpha}$ by $B_{r, \alpha}=\left\{f: f^{(r-1)} \in C[0, \infty), f_{ \pm}^{(r)}(x)\right.$ exist everywhere and are bounded on every finite subinterval of $[0, \infty)$ and $f_{ \pm}^{(r)}=O\left(e^{\alpha t}\right)(t \rightarrow \infty)$, for some $\left.\alpha>0\right\}$, $r=1,2, \ldots$. By $f_{ \pm}^{(0)}(x)$ we mean $f(x \pm)$.

Now we are ready to prove and state our main theorem.

Theorem 3.1. Let $f \in B_{r, \alpha}, r=1,2, \ldots, \alpha>0$. Then for every $x \in(0, \infty)$ and $n \geq \max \{2,4 \alpha\}$,

$$
\begin{aligned}
& \left|P_{n}^{(r)}(f, x)-\frac{1}{2}\left\{f_{+}^{(r)}(x)+f_{-}^{(r)}(x)\right\}\right| \\
& \quad \leq \frac{|2 r-1|}{\sqrt{8 e n x}} \cdot\left|f_{+}^{(r)}(x)-f_{-}^{(r)}(x)\right|+\frac{x+2 \lambda}{n x} \sum_{k=1}^{n} V_{x-x / \sqrt{k}}^{x+x / \sqrt{k}}\left(g_{r, x}\right)+(n x)^{-1 / 2}\left(\lambda 2^{r}\right)^{1 / 2} e^{2 \alpha x},
\end{aligned}
$$

where $g_{r, x}$ is the auxiliary function defined by

$$
g_{r, x}(t)= \begin{cases}f^{(r)}(t)-f_{-}^{(r)}(x), & 0 \leq t<x, \\ 0, & t=x, \\ f^{(r)}(t)-f_{+}^{(r)}(x), & x<t<\infty,\end{cases}
$$

and $V_{a}^{b}\left(g_{r, x}(t)\right)$ is the total variation of $g_{r, x}(t)$ on $[a, b]$. In particular $g_{0, x}(t) \equiv g_{x}(t)$ as defined in [3]. 
6 Simultaneous approximation for the Phillips operators

Proof. Clearly

$$
\begin{aligned}
& \left|P_{n}^{(r)}(f, x)-\frac{1}{2}\left\{f_{+}^{(r)}(x)+f_{-}^{(r)}(x)\right\}\right| \\
& \quad \leq\left|P_{n}^{(r)}\left(g_{r, x}(t), x\right)\right|+\frac{1}{2}\left|f_{+}^{(r)}(x)-f_{-}^{(r)}(x)\right| \cdot\left|P_{n}^{(r)}(\operatorname{sign}(t-x), x)\right| .
\end{aligned}
$$

In order to prove the result we need the estimates for $P_{n}^{(r)}\left(g_{r, x}, x\right)$ and $P_{n}^{(r)}(\operatorname{sign}(t-x), x)$.

We first estimate $P_{n}^{(r)}(\operatorname{sign}(t-x), x)$ as follows:

$$
\begin{aligned}
P_{n}^{(r)}(\operatorname{sign}(t-x), x) & =\int_{x}^{\infty} K_{r, n}(x, t) d t-\int_{0}^{x} K_{r, n}(x, t) d t \\
& =A_{r, n}(x)-B_{r, n}(x), \quad \text { say. }
\end{aligned}
$$

It is easily verified that $A_{r, n}(x)+B_{r, n}(x)=1$. Thus $P_{n}^{(r)}(\operatorname{sign}(t-x), x)=1-2 A_{r, n}(x)$.

Using the fact that $\sum_{j=0}^{k+r-1} p_{n, j}(x)=n \int_{x}^{\infty} p_{n, k+r-1}(t) d t$, we get

$$
\begin{aligned}
A_{r, n}(x) & =n \sum_{k=0}^{\infty} p_{n, k}(x) \int_{x}^{\infty} p_{n, k+r-1}(t) d t=\sum_{k=0}^{\infty} p_{n, k}(x) \sum_{j=0}^{k+r-1} p_{n, j}(x) \\
& =\sum_{k=0}^{\infty} p_{n, k}(x)\left(\sum_{j=0}^{k} p_{n, j}(x)+\sum_{j=k+1}^{k+r-1} p_{n, j}(x)\right) \\
& \leq \sum_{k=0}^{\infty} p_{n, k}(x) \sum_{j=0}^{k} p_{n, j}(x)+\frac{r-1}{\sqrt{2 e n x}} .
\end{aligned}
$$

Proceeding along similar lines as in [3], we get

$$
\left|A_{r, n}(x)-B_{r, n}(x)\right|=\left|2 A_{r, n}(x)-1\right| \leq \frac{|2 r-1|}{\sqrt{2 e n x}} .
$$

Next we estimate $P_{n}^{(r)}\left(g_{r, x}, x\right)$, and for this, note that by Lebesgue-Stieltjes integral representation, we have

$$
\begin{aligned}
P_{n}^{(r)}\left(g_{r, x}, x\right) & =\int_{0}^{\infty} g_{r, x}(t) K_{r, n}(x, t) d t=\left(\int_{I_{1}}+\int_{I_{2}}+\int_{I_{3}}+\int_{I_{4}}\right) K_{r, n}(x, t) g_{r, x}(t) d t \\
& =R_{1}+R_{2}+R_{3}+R_{4}, \quad \text { say, }
\end{aligned}
$$

where $I_{1}=[0, x-x / \sqrt{n}], I_{2}=[x-x / \sqrt{n}, x+x / \sqrt{n}], I_{3}=[x+x / \sqrt{n}, 2 x]$, and $I_{4}=[2 x, \infty)$. Let us define $\eta_{r, n}(x, t)=\int_{0}^{t} K_{r, n}(x, u) d u$.

We first estimate $R_{1}$, and for this if we write $y=x-x / \sqrt{n}$ and use integration by parts, we get

$$
R_{1}=\int_{0}^{y} g_{r, x}(t) d_{t}\left(\eta_{r, n}(x, t)\right)=g_{r, x}(y) \eta_{r, n}(x, t)-\int_{0}^{y} \eta_{r, n}(x, t) d_{t}\left(g_{r, x}(t)\right)
$$


By Remark 2.5, it follows that

$$
\begin{aligned}
\left|R_{1}\right| & \leq V_{y}^{x}\left(g_{r, x}\right) \eta_{r, n}(x, y)+\int_{0}^{y} \eta_{r, n}(x, t) d_{t}\left(-V_{t}^{x}\left(g_{r, x}\right)\right) \\
& \leq V_{y}^{x}\left(g_{r, x}\right) \frac{\lambda x}{n(x-y)^{2}}+\frac{\lambda x}{n} \int_{0}^{y} \frac{1}{(x-t)^{2}} d_{t}\left(-V_{t}^{x}\left(g_{r, x}\right)\right) .
\end{aligned}
$$

Integrating by parts the last term, we get after simple computation

$$
\left|R_{1}\right| \leq \frac{\lambda x}{n}\left[\frac{V_{0}^{x}\left(g_{r, x}\right)}{x^{2}}+2 \int_{0}^{y} \frac{V_{t}^{x}\left(g_{r, x}\right)}{(x-t)^{3}} d t\right] .
$$

Now replacing the variable $y$ in the last integral by $x-x / \sqrt{t}$, we get

$$
\left|R_{1}\right| \leq \frac{2 \lambda}{n x} \sum_{k=1}^{n} V_{x-x / \sqrt{k}}^{x}\left(g_{r, x}\right) .
$$

Next, we estimate $R_{2}$, and for this, note that for $t \in[x-x / \sqrt{n}, x+x / \sqrt{n}]$, we have

$$
\left|g_{r, x}(t)\right|=\left|g_{r, x}(t)-g_{r, x}(x)\right| \leq V_{x-x / \sqrt{n}}^{x+x / \sqrt{n}}\left(g_{r, x}\right) .
$$

Also, by the fact that $\int_{a}^{b} d_{t}\left(\eta_{r, n}(x, t)\right) \leq 1$ for $(a, b) \subset[0, \infty)$, we get

$$
\left|R_{2}\right| \leq V_{x-x / \sqrt{n}}^{x+x / \sqrt{n}}\left(g_{r, x}\right) \leq \frac{1}{n} \sum_{k=1}^{n} V_{x-x / \sqrt{k}}^{x+x / \sqrt{k}}\left(g_{r, x}\right) .
$$

Now to estimate $R_{3}$, write $z=x+x / \sqrt{n}$. Then

$$
\begin{aligned}
R_{3} & =\int_{z}^{2 x} K_{r, n}(x, t) g_{r, x}(t) d t=-\int_{z}^{2 x} g_{r, x}(t) d_{t}\left(1-\eta_{r, n}(x, t)\right) \\
& =-g_{r, x}(2 x)\left(1-\eta_{r, n}(x, 2 x)\right)+g_{r, x}(z)\left(1-\eta_{r, n}(x, z)\right)+\int_{z}^{2 x}\left(1-\eta_{r, n}(x, t)\right) d_{t} g_{r, x}(t) .
\end{aligned}
$$

Since $\left|g_{r, x}(t)\right|=\left|g_{r, x}(t)-g_{r, x}(x)\right| \leq V_{x}^{t}\left(g_{r, x}\right)$, it follows by Lemma 2.7 that

$$
\left|R_{3}\right| \leq \frac{\lambda x}{n}\left\{x^{-2} V_{x}^{2 x}\left(g_{r, x}\right)+(z-x)^{-2} V_{x}^{z}\left(g_{r, x}\right)+\int_{z}^{2 x}(t-x)^{-2} d_{t} V_{x}^{t}\left(g_{r, x}\right)\right\} .
$$

Again integrating by parts, we get

$$
\left|R_{3}\right| \leq \frac{\lambda x}{n}\left\{2 x^{-2} V_{x}^{2 x}\left(g_{r, x}\right)+2 \int_{z}^{2 x} V_{x}^{t}\left(g_{r, x}\right)(t-x)^{-3} d t\right\} .
$$

Arguing similarly as in the estimate of $R_{1}$, we obtain

$$
\left|R_{3}\right| \leq \frac{2 \lambda}{n x} \sum_{k=1}^{n} V_{x}^{x+x / \sqrt{k}}\left(g_{r, x}\right) .
$$


8 Simultaneous approximation for the Phillips operators

Finally, we estimate $R_{4}$ as follows:

$$
\begin{aligned}
\left|R_{4}\right| & =\left|\int_{2 x}^{\infty} K_{r, n}(x, t) g_{r, x}(t) d t\right| \leq n \sum_{k=0}^{\infty} p_{n, k}(x) \int_{2 x}^{\infty} p_{n, k+r-1}(t) e^{\alpha \cdot t} d t \\
& \leq \frac{n}{x} \sum_{k=0}^{\infty} p_{n, k}(x) \int_{0}^{\infty} p_{n, k+r-1}(t) e^{\alpha \cdot t}|t-x| d t \\
& \leq \frac{1}{x}\left(n \sum_{k=0}^{\infty} p_{n, k}(x) \int_{0}^{\infty} p_{n, k+r-1}(t)(t-x)^{2} d t\right)^{1 / 2}\left(n \sum_{k=0}^{\infty} p_{n, k}(x) \int_{0}^{\infty} p_{n, k+r-1}(t) e^{2 \alpha \cdot t} d t\right)^{1 / 2} .
\end{aligned}
$$

To estimate the above first expression we will use Remark 2.5, and to evaluate the second expression, we note that

$$
\begin{aligned}
n \sum_{k=0}^{\infty} p_{n, k}(x) \int_{0}^{\infty} p_{n, k+r-1}(t) e^{2 \alpha \cdot t} d t \\
\quad=n \sum_{k=0}^{\infty} p_{n, k}(x) \frac{n^{k+r-1}}{(k+r-1) !} \int_{0}^{\infty} t^{k+r-1} e^{-(n-2 \alpha) t} d t \\
\quad=n \sum_{k=0}^{\infty} p_{n, k}(x) \frac{n^{k+r-1}}{(k+r-1) !} \frac{\Gamma(k+r)}{(n-2 \alpha)^{k+r}}=\frac{n^{r}}{(n-2 \alpha)^{r}} \sum_{k=0}^{\infty}\left(\frac{n}{n-2 \alpha}\right)^{k} p_{n, k}(x) \\
=\frac{n^{r}}{(n-2 \alpha)^{r}} e^{-n x} \sum_{k=0}^{\infty}\left(\frac{n^{2} x}{n-2 \alpha}\right)^{k} \frac{1}{k !}=\frac{n^{r}}{(n-2 \alpha)^{r}} e^{2 n x \alpha /(n-2 \alpha)} \leq 2^{r} e^{4 \alpha x} \quad \text { for } n>4 \alpha .
\end{aligned}
$$

If we now combine the above estimate with Remark 2.5, we get

$$
\begin{aligned}
\left|R_{4}\right| & \leq \frac{1}{x}\left(n \sum_{k=0}^{\infty} p_{n, k}(x) \int_{0}^{\infty} p_{n, k+r-1}(t)(t-x)^{2} d t\right)^{1 / 2}\left(n \sum_{k=0}^{\infty} p_{n, k}(x) \int_{0}^{\infty} p_{n, k+r-1}(t) e^{2 \alpha \cdot t} d t\right)^{1 / 2} \\
& \leq(n x)^{-1 / 2}\left(\lambda 2^{r}\right)^{1 / 2} e^{2 \alpha x} .
\end{aligned}
$$

Finally, combining the estimates obtained in (3.3)-(3.20), we get the required result, and the proof of the theorem is thus complete.

Remark 3.2. Since the Bézier variant of the Phillips operators for $\beta \geq 1$ is defined by

$$
P_{n, \beta}(f, x)=n \sum_{k=1}^{\infty} Q_{n, k}^{(\beta)}(x) \int_{0}^{\infty} p_{n, k-1}(t) f(t) d t+Q_{n, 0}^{(\beta)}(x) f(0),
$$

where $Q_{n, k}^{(\beta)}(x)=J_{n, k}^{\beta}(x)-J_{n, k+1}^{\beta}(x), J_{n, k}(x)=\sum_{i=k}^{\infty} p_{n, i}(x)$, the rate of convergence in simultaneous approximation for the above Bézier variant of Phillips operators can be obtained along similar lines. We omit the details. 


\section{Acknowledgment}

This research was carried out while the second author was visiting Auburn University, USA, during the fall of 2005.

\section{References}

[1] Z. Finta and V. Gupta, Direct and inverse estimates for Phillips type operators, Journal of Mathematical Analysis and Applications 303 (2005), no. 2, 627-642.

[2] V. Gupta and R. P. Pant, Rate of convergence for the modified Szász-Mirakyan operators on functions of bounded variation, Journal of Mathematical Analysis and Applications 233 (1999), no. 2, 476-483.

[3] V. Gupta and G. S. Srivastava, On the rate of convergence of Phillips operators for functions of bounded variation, Annales Societatis Mathematicae Polonae. Seria I. Commentationes Mathematicae 36 (1996), 123-130.

[4] C. P. May, On Phillips operator, Journal of Approximation Theory 20 (1977), no. 4, 315-332.

[5] R. S. Phillips, An inversion formula for Laplace transforms and semi-groups of linear operators, Annals of Mathematics. Second Series 59 (1954), 325-356.

[6] H. M. Srivastava and V. Gupta, A certain family of summation-integral type operators, Mathematical and Computer Modelling 37 (2003), no. 12-13, 1307-1315.

[7] H. M. Srivastava and X.-M. Zeng, Approximation by means of the Szász-Bézier integral operators, International Journal of Pure and Applied Mathematics 14 (2004), no. 3, 283-294.

[8] X.-M. Zeng and J.-N. Zhao, Exact bounds for some basis functions of approximation operators, Journal of Inequalities \& Applications 6 (2001), no. 5, 563-575.

N. K. Govil: Department of Mathematics and Statistics, Auburn University, Auburn,

AL 36849-5108, USA

E-mail address: govilnk@auburn.edu

Vijay Gupta: School of Applied Sciences, Netaji Subhas Institute of Technology, Sector 3 Dwarka, New Delhi 110075, India

E-mail address: vijay@nsit.ac.in

Muhammad Aslam Noor: Mathematics Department, COMSATS Institute of Information Technology, Islamabad, Pakistan

E-mail address: aslamnoor@comsats.edu.pk 


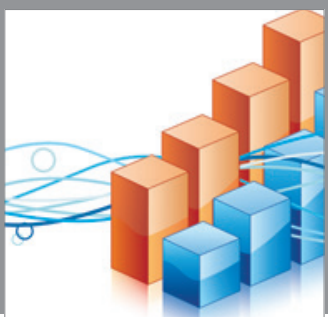

Advances in

Operations Research

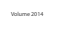

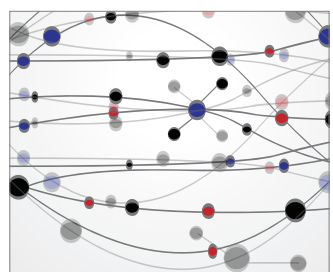

\section{The Scientific} World Journal
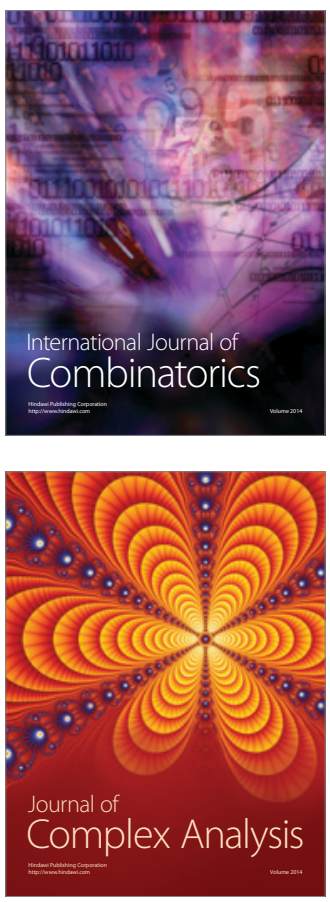

International Journal of

Mathematics and

Mathematical

Sciences
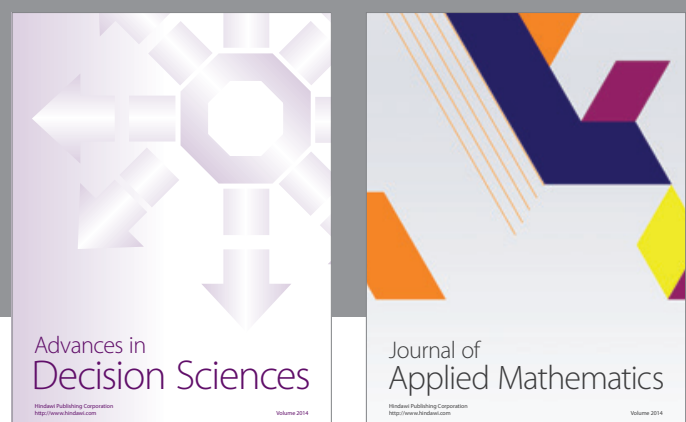

Journal of

Applied Mathematics
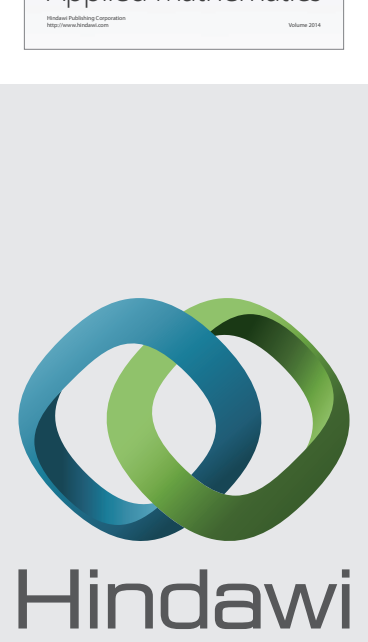

Submit your manuscripts at http://www.hindawi.com
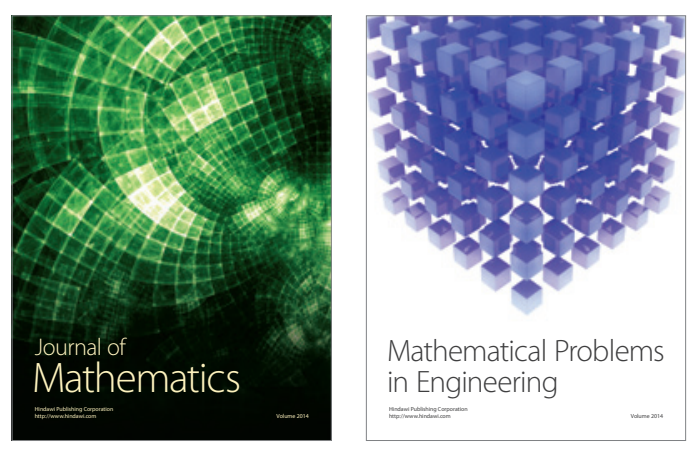

Mathematical Problems in Engineering
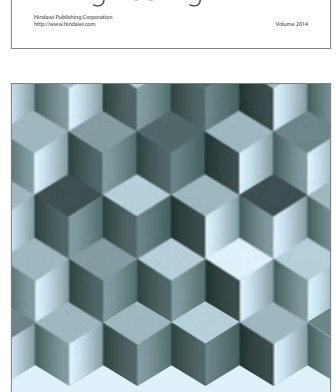

Journal of

Function Spaces
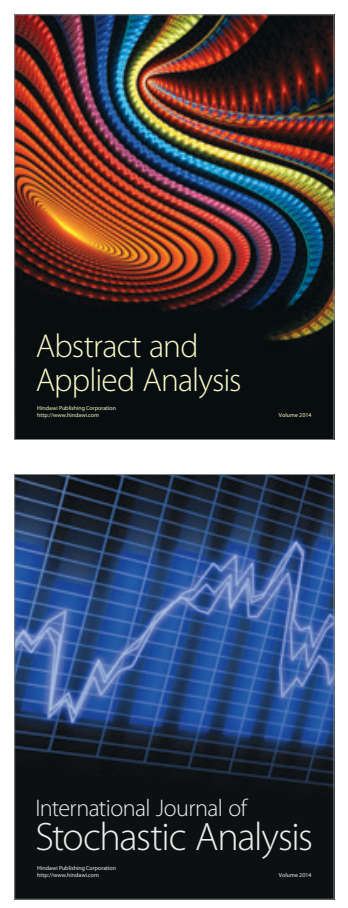

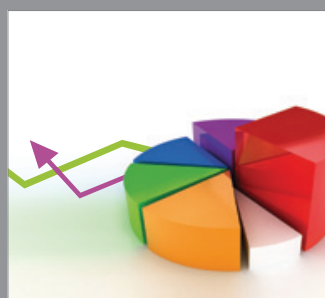

ournal of

Probability and Statistics

Promensencen
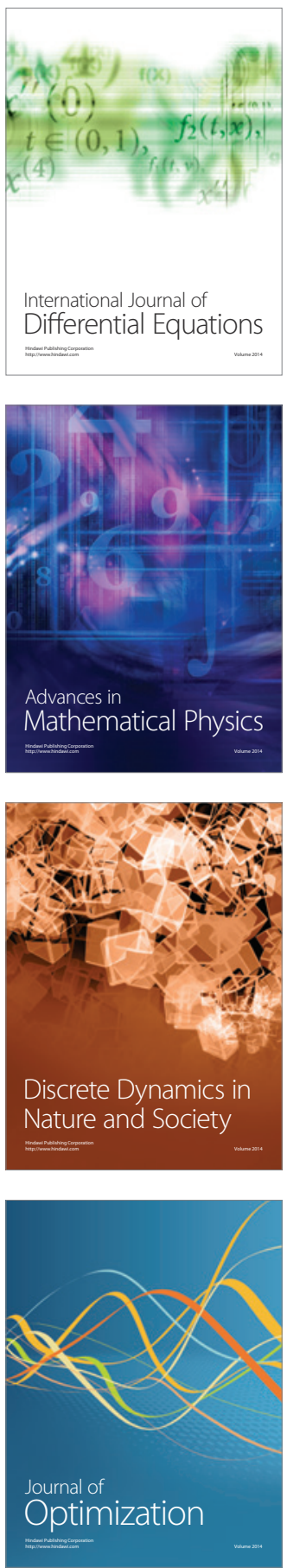